\title{
Artrodesi circonferenziale per frattura di T11: approccio combinato posteriore e anteriore in toracoscopia. Caso clinico
}

A. Barbanera, M.Vitali, A. Bertuccio, P.P. Versari

SOC Neurochirurgia, Azienda Ospedaliera SS. Antonio e Biagio e C. Arrigo, Alessandria

\begin{abstract}
Circonferential arthrodesis for treatment of T11 fracture: combined posterior and anterior thoracoscopic approach. Case repor

We present a case report of a T11 (type C, according to Magerl classification) fracture treated by a combined posterior and anterior thoracoscopic approach. This surgical choice allows to obtain an optimal primary stability and arthrodesis, with a short posterior stabilization. Thoracoscopy avoids the invasiveness of the anterior approach.
\end{abstract}

\section{Caso clinico}

Una donna di 45 anni, in seguito a incidente stradale, riportava un trauma toraco-addominale. Una TC "total body" escludeva lesioni viscerali e documentava una frattura di T11 di tipo C secondo la classificazione di Magerl (Fig. 1). L'obiettività neurologica all'ingresso in reparto risultava nella norma. In considerazione del tipo di frattura, si è deciso di effettuare un intervento combinato: un approccio posteriore (posizionamento di viti peduncolari in T10 e T12), un approccio anteriore in toracoscopia (corpectomia di T11, posizionamento di "cage" riempita di osso autologo,

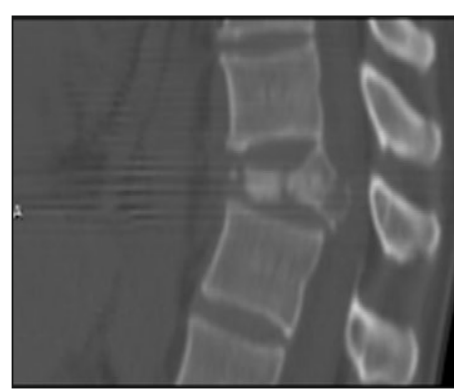

Fig. 1. Ricostruzione TC sagittale della frattura di T11: frattura a scoppio del soma con associata cifosi

correzione della cifosi e posizionamento di placca tra T10 e T12) seguiti da un terzo tempo posteriore di serraggio del sistema di stabilizzazione e artrodesi poste-

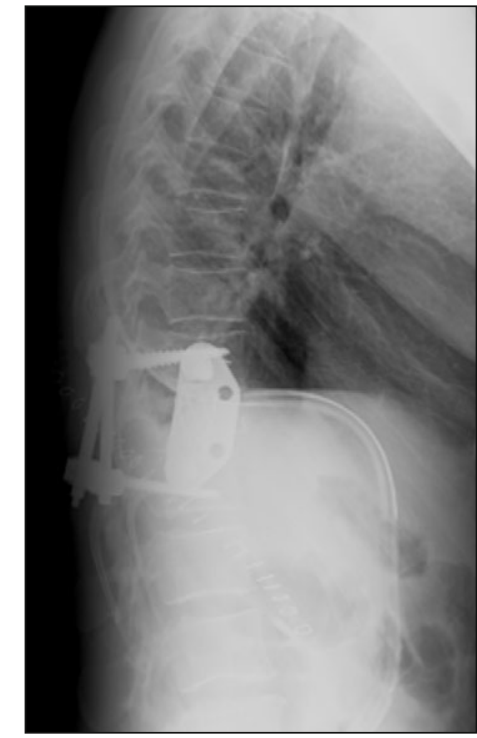

Fig. 2. Controllo post-operatorio: stabilizzazione posteriore con viti peduncolari in T10 e T12 connesse da barre; "cage" intersomatica riempita di osso autologo con placca laterale

ro-laterale sempre con osso autologo.

Controlli radiologici post-operatori hanno mostrato una buona correzione della cifosi, con buon allineamento metamerico e ottima

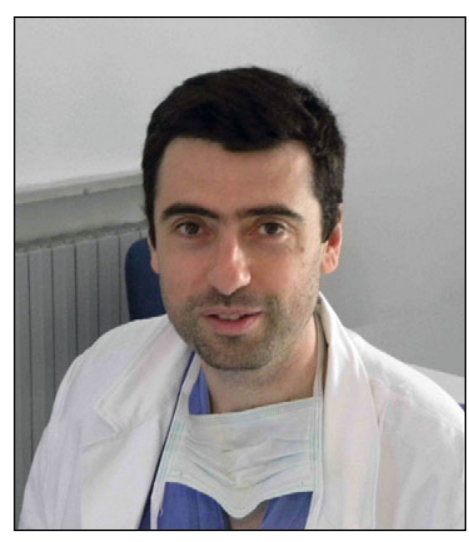

A. Barbanera

Per fratture di tipo C una tale scelta chirurgica garantisce una perfetta stabilità primaria, permettendo di agire direttamente sulle colonne anteriore e posteriore interessate dal trauma, offre la garanzia di una ottimale artrodesi a distanza e di una buona correzione della deformità post-traumatica. L'utilizzo della toracoscopia per il tempo anteriore minimizza la perdita ematica, le complicanze strettamente connesse alla toracotomia, il dolore post-operatorio e il danno estetico. Dopo una adeguata curva di apprendimento i tempi operatori non risultano allungati dalla scelta della toracoscopia. Gli approcci combinati anteriori-posteriori offrono infine la possibilità di avvalersi di una stabilizzazione posteriore corta, limitata in questo caso ai livelli immediatamente adiacenti alla frattura.

\section{Letture consigliate}

Aebi M (2010) Classification of thoracolumbar fractures and dislo-
$1] \cdot 2-7$ al (2004) Thoracoscopic placement of dual-rod instrumentation in thoracic spinal trauma. Neurosurgery 54:1150-1153

Khoo LT, Beisse R, Potulski M (2002)

Thoracoscopic-assisted treatment of thoracic and lumbar fractures: a series of 371 consecutive cases. Neurosurgery 51[5 Suppl.]:104117

Kim DH, Jaikumar S, Kam AC (2002) Minimally invasive spine instrumentation. Neurosurgery 51[5 Suppl.]:15-25

Lekovic GP, Horn EM, Dickman CA (2006) Distraction injury to thoracic spine treated with thoracoscopic dual-rod fixation. Spine J 6:330-334

Magerl F, Aebi M, Gertzbein SD et al (1994) A comprehensive classification of thoracic and lumbar injuries. Eur Spine J 3:184-201 